\title{
Studies on the interleukin-10 gene in animal models of colitis
}

\author{
Hugh J Freeman MD
}

\begin{abstract}
ARTICLE
Barbara G, Xing Z, Hogaboam CM, Gauldie J, Collins SM. Interleukin 10 gene transfer prevents experimental colitis in rats. Gut 2000;46:344-9.

Madsen KL, Doyle JS, Tavernini MM, Jewell LD, Rennie RP, Fedorak RN. Antibiotic therapy attenuates colitis in interleukin 10 gene-deficient mice. Gastroenterology 2000;118:1084-105.
\end{abstract}

\section{BACKGROUND}

Cytokines play a role in the inflammatory process in colitis and may have therapeutic potential. Interleukin-10 (IL-10) has both immunomodulatory and anti-inflammatory properties. IL-10-deficient mice develop intestinal inflammation with increased tissue levels of other cytokines, including tumour necrosis factor-alpha. In patients with inflammatory bowel disease, impaired IL-10 production by lamina propria $\mathrm{T}$ cells occurs and human recombinant IL-10 improves clinical parameters in inflammatory bowel disease (eg, Crohn's disease). There seem to be conflicting results in differing animal models, and the timing of administration of IL-10 relative to onset of colitis may be critical, possibly due to rapid clearance of IL-10. Interestingly, in IL-10 gene-defi- cient mice raised in germ-free conditions, the intestinal inflammatory changes normally observed in conventional nongerm-free conditions are not detected, suggesting a role for luminal bacteria in the pathogenesis of the inflammatory process.

Barbara et al from McMaster University in Hamilton, Ontario evaluated gene transfer with an adenovirus IL-10 construct in IL-10-deficient mice for the treatment of colitis. Madsen et al from the University of Alberta in Edmonton, Alberta evaluated antibiotic effects in IL-10deficient mice on intestinal histopathology, along with alterations in luminal surface bacterial flora. While these animal models have limited applicability to human disease, some observations on the hypothetical role of IL-10 in the intestinal inflammatory process were described.

\section{RESEARCH STUDIES}

In the study by Barbara et al, a chemically induced (dinitrobenzene $[\mathrm{DNB}])$ rat colitis model was used. A humantype adenovirus with IL-10 gene was injected intraperitoneally before and after DNB induction. Colon damage was evaluated with descriptive pathological methods, myeloperoxidase activity and leukotriene $\mathrm{B}_{4}$ concentrations using standard laboratory assays. Pathological end points included macroscopic evaluation at laparotomy, including the gross appearance of the colonic wall and the colonic mucosa along with light microscopic examination 
using hematoxylin-stained sections. Two observers blinded to the treatment regimens recorded the pathological findings. This study showed that gene transfer before the induction of DNB rat intestinal inflammation increased the IL-10 concentration and improved the colitis (pathology, myeloperoxidase activities and leukotriene $\mathrm{B}_{4}$ concentrations), but that gene transfer after DNB treatment had no detectable beneficial effect on the end points examined.

In the study by Madsen et al, IL-10 gene-deficient mice were evaluated with histological scoring of small and large intestinal sections done independently by two pathologists. Colonic luminal bacteria were enumerated along with speciation under anaerobic conditions with colonic tissue homogenates using standard microbiological methods. To investigate the role of bacteria further in the initiation and perpetuation of inflammation, different antibiotic regimens were given to nursing mothers and, after weaning, in drinking water: ciprofloxacin for luminal Gram-negative bacteria and neomycin with metronidazole for colonic anaerobes. Longitudinal evaluations were done before and after histological evidence of the onset of colitis. This study showed that, before having colitis, IL-10-deficient mice had more bacteria adherent to the colonic mucosa. Treatment with either antibiotic regimen reduced the adherent bacteria and prevented colitis (as reflected in scoring).

Differentiation of antibiotic regimens showed that neomycin and metronidazole were more efficacious than ciprofloxacin in treating established colitis.

\section{DISCUSSION}

Although both studies assessed the relationship of colitis in animal models and a possible critical role of IL-10, it is not known whether there is a common thread or parallel with any of the human forms of inflammatory bowel disease. Direct extrapolation of these results from differing animal models of colitis to any form of human intestinal inflammatory disease remains difficult.

In the first study, IL-10 gene transfer was successful in apparently preventing, but not in reversing, rat colitis induced by an external chemical agent, DNB. While this study illustrated that gene transfer could prevent at least a particular type of chemically induced experimental colitis, benefit could not be defined once this particular type of colitis was established. The real significance of this study may be indirect (because no exogenous agent is known to induce chronic inflammatory bowel disease in humans). The timing of IL-10 administration as a possible treatment regimen in humans may be critical. Future studies using recombinant IL-10 in humans may need to consider this issue, particularly if the definition of genetically related and predisposed individuals were defined before the onset of inflammatory bowel disease.

In the second study, the importance of intestinal luminal bacterial populations was explored in an IL-10 gene-deficient mouse model of colitis. In this model, elimination or reduction of some luminal or adherent species of bacteria appeared to play an important role in the pathogenesis of the colitis. This study provides additional data in a genetically defined animal model that qualitative and quantitative perturbations in the luminal microflora may be very important in the initiation as well as the perpetuation of the intestinal inflammatory process. Future studies, especially those related to the ongoing search for effective forms of therapy, will need to focus attention not only on the inflamed intestine, but also on critically significant events occurring within the intestinal lumen. 


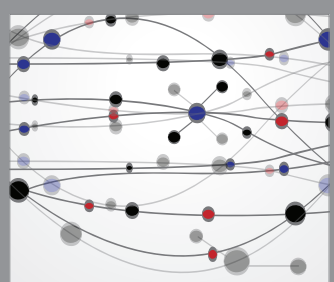

The Scientific World Journal
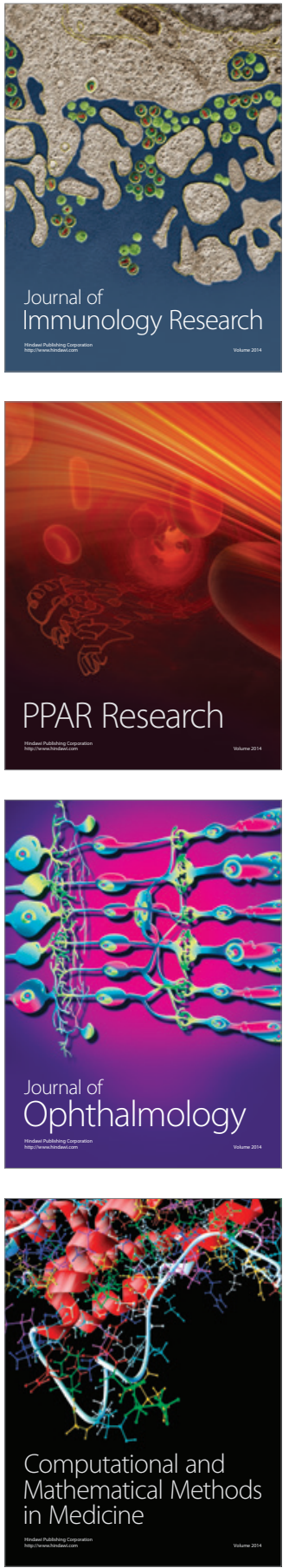

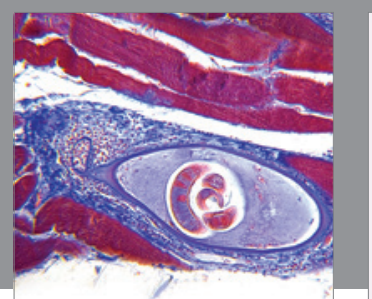

Gastroenterology Research and Practice

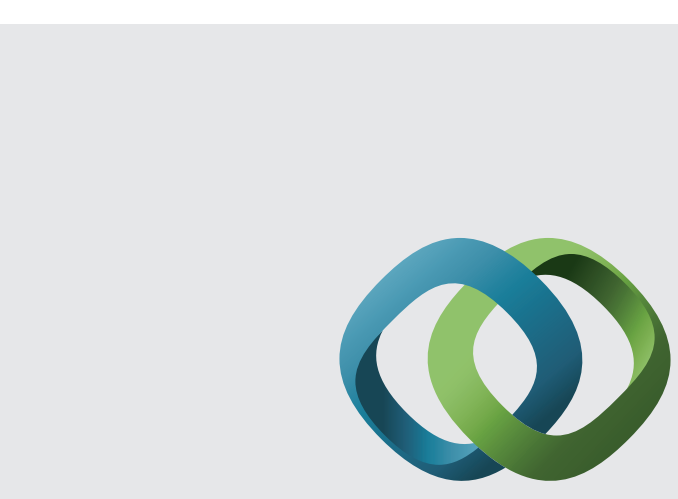

\section{Hindawi}

Submit your manuscripts at

http://www.hindawi.com
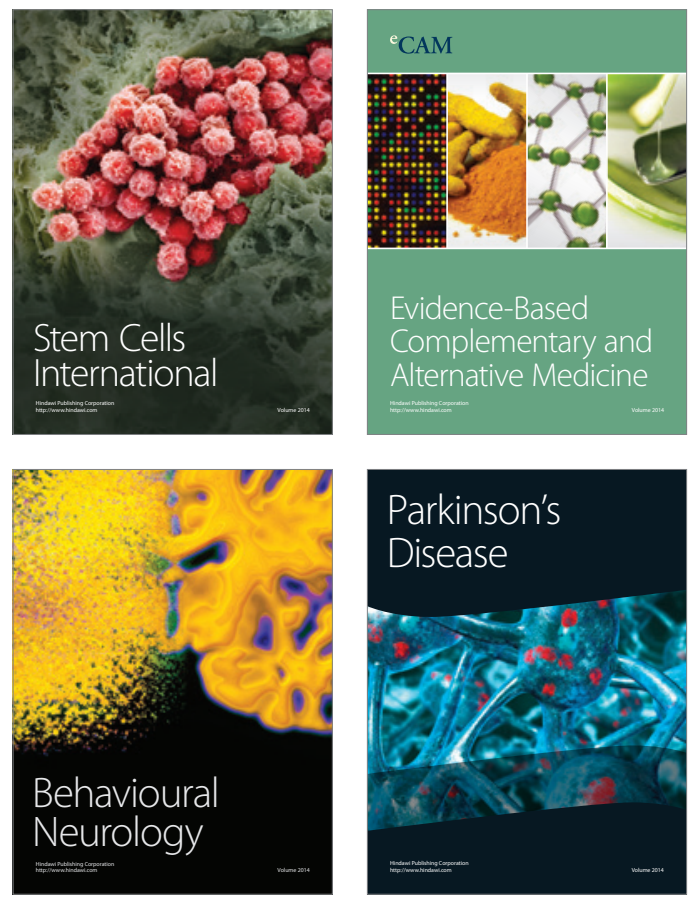
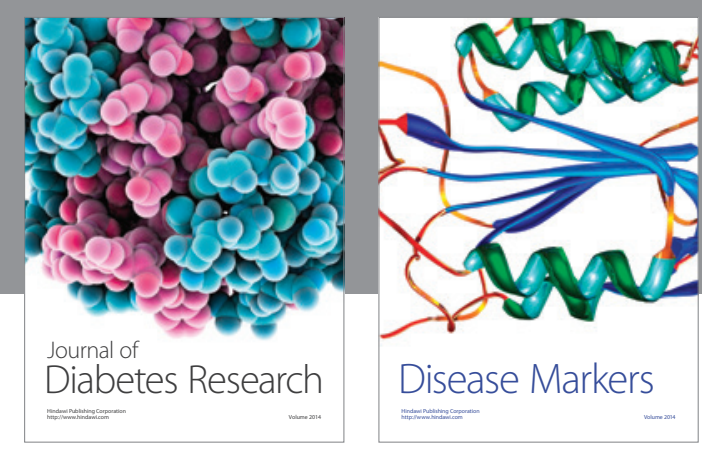

Disease Markers
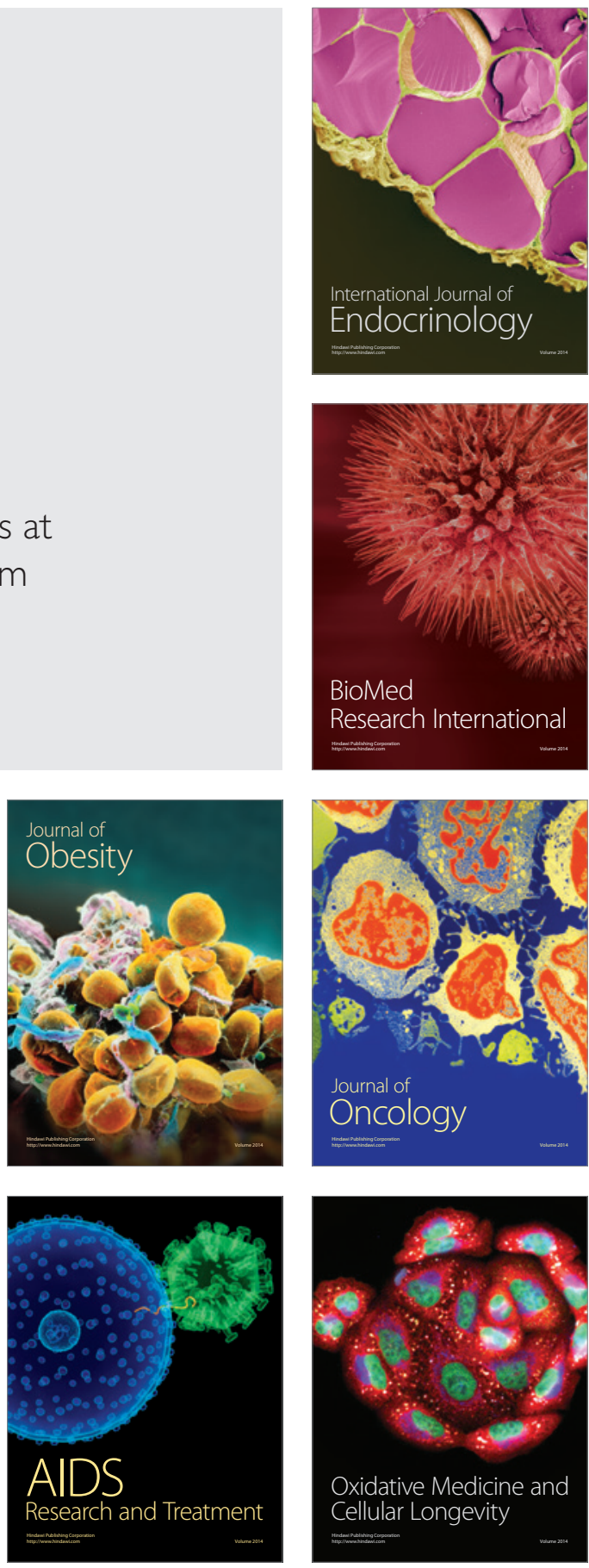